\title{
Qubit dynamics in a $q$-deformed oscillators environment
}

\author{
Sonia L'Innocente, ${ }^{1}$ Cosmo Lupo, ${ }^{2}$ and Stefano Mancini ${ }^{2,3}$ \\ ${ }^{1}$ Dipartimento di Matematica \& Informatica, Università di Camerino, I-62032 Camerino, Italy \\ ${ }^{2}$ Dipartimento di Fisica, Università di Camerino, I-62032 Camerino, Italy \\ ${ }^{3}$ INFN, Sezione di Perugia, I-06123 Perugia, Italy
}

\begin{abstract}
We study the dynamics of one and two qubits plunged in a $q$-deformed oscillators environment. Specifically we evaluate the decay of quantum coherence and entanglement in time when passing from bosonic to fermionic environments. Slowing down of decoherence in the fermionic case is found. The effect only manifests at finite temperature.
\end{abstract}

\section{INTRODUCTION}

Open system dynamics is of uppermost importance in the quantum regime where non classical phenomena turn out to be very fragile with respect to any noise source. The noise effects are often modeled as the result of an interaction of the system with a large number of uncontrollable degrees of freedom, i.e. an environment [1]. Environments can be assumed as to be composed by different kinds of particles, for instance oscillators or spin- $\frac{1}{2}$. These objects come, under the mathematical point of view, from the realizations of two different algebras (the Heisenberg-Weyl algebra and the Lie algebra su(2)) corresponding to fermionic and bosonic commutation relations. These latter can be seen as two limit cases of more general commutation relations involving deformed algebras parameterized by one continuous parameter [2, 3, 4].

Our aim is to analyze the qubit dynamics in an environment of oscillators satisfying suitable $q$-deformed commutation relations, such that it permits to continuously interpolate between oscillators and spin- $\frac{1}{2}$. Actually, we investigate how quantum decoherence phenomena changes in passing from bosonic to fermionic environments. We find a slowing down of decoherence in the fermionic case. However, this effect only manifests at finite temperature.

The paper is organized as follows. In Section [I] we present the model. We then derive the master equation in Section [III In Section [V] we study the dynamics of a single qubit and we evaluate its coherence decay. We then study the dynamics of two qubits and we evaluate the entanglement decay by distinguishing the case of the two qubits in the same environment (Section V), from that of the two qubits in separate environments (Section VI). Finally, Section VII is for concluding remarks.

\section{THE MODEL}

Let us consider a system (qubit) described by the free Hamiltonian

$$
H_{S}=\Omega \sigma_{z},
$$

with $\Omega$ the qubit frequency and $\sigma, \sigma^{\dagger}, \sigma_{z}$ operators satisfying the commutation relations

$$
\begin{aligned}
{\left[\sigma^{\dagger}, \sigma\right] } & =\sigma_{z}, \\
{\left[\sigma, \sigma_{z}\right] } & =2 \sigma, \\
{\left[\sigma^{\dagger}, \sigma_{z}\right] } & =-2 \sigma^{\dagger} .
\end{aligned}
$$

They define the su(2) algebra. Furthermore, we consider an environment composed by an infinite (countable) number of oscillators whose Hamiltonian reads as [5, 6]

$$
H_{E}=\sum_{k} \omega_{k} N_{k}
$$

with $\omega_{k}$ the frequency of the $k$-th oscillator and $A_{k}, A_{k}^{\dagger}, N_{k}$ operators satisfying the commutation relations

$$
\begin{aligned}
& {\left[N_{h}, A_{k}\right]=-\delta_{h k} A_{k},} \\
& {\left[N_{h}, A_{k}^{\dagger}\right]=\delta_{h k} A_{k}^{\dagger} .}
\end{aligned}
$$

They define the Heisenberg-Weyl algebra. We are now going to introduce a deformation of this algebra through the so-called "quons" commutation relations [5]

$$
A_{h} A_{k}^{\dagger}-q A_{k}^{\dagger} A_{h}=\delta_{h k},
$$


where $q \in[-1,1]$ is the deformation parameter. It allows us to interpolate between fermions $(q=-1)$ and bosons $(q=1)$. Intermediate values of $q \in(-1,1)$ correspond to the so-called "infinite statistics".

We assume the system interacting with the environment through the following Hamiltonian

$$
H_{I}=\sum_{k} \lambda_{k}\left(A_{k}^{\dagger} \sigma+A_{k} \sigma^{\dagger}\right)
$$

where $\lambda_{k}$ denotes the coupling constant of the system with the $k$-th environment's oscillator.

\section{MASTER EQUATION}

Quite generally, the master equation for the system density operator $\rho$ can be derived by using the Born-Markov approximation [1]. Hence, it can be formally written as

$$
\dot{\rho}(t)=-\int_{0}^{\infty} d \tau \operatorname{Tr}_{E}\left\{\left[H_{I}(t),\left[H_{I}(t-\tau), \rho(t) \otimes \rho_{E}\right]\right]\right\},
$$

where $\rho_{E}$ is the initial environment density operator and $\operatorname{Tr}_{E}$ denotes the trace over environment degrees of freedom. Furthermore, it is

$$
H_{I}(t)=e^{\iota\left(H_{S}+H_{E}\right) t} H_{I} e^{-\iota\left(H_{S}+H_{E}\right) t} .
$$

For the choice of the environment Hamiltonian (5), the dynamical equations are formally identical to the undeformed case. The reason is that the interaction Hamiltonian $H_{I}(t)$ reads as follows

$$
H_{I}(t)=\sum_{k} \lambda_{k}\left(A_{k}^{\dagger} \sigma e^{-\iota\left(\omega_{k}-\Omega\right) t}+A_{k} \sigma^{\dagger} e^{\iota\left(\omega_{k}-\Omega\right) t}\right),
$$

by virtue of (11), (9), (5) and (1). Therefore, from (10), we can write

$$
\dot{\rho}(t)=-\int_{0}^{\infty} d \tau \operatorname{Tr}_{E} \sum_{k, l} \lambda_{k} \lambda_{l} \mathcal{F}_{k l}(\rho(t)),
$$

where

$$
\begin{aligned}
\mathcal{F}_{k l}(\rho(t))= & F_{k}(t) F_{l}(t-\tau) \rho(t) \otimes \rho_{E}-F_{k}(t) \rho(t) \otimes \rho_{E} F_{l}(t-\tau) \\
& -F_{k}(t-\tau) \rho(t) \otimes \rho_{E} F_{l}(t)+\rho(t) \otimes \rho_{E} F_{k}(t-\tau) F_{l}(t)
\end{aligned}
$$

and

$$
F_{k}(t)=A_{k}^{\dagger} \sigma e^{-\iota\left(\omega_{k}-\Omega\right) t}+A_{k} \sigma^{\dagger} e^{\iota\left(\omega_{k}-\Omega\right) t} .
$$

We now assume an initial thermal state for the environment at temperature $T$,

$$
\rho_{E}=Z^{-1} e^{-H_{E} / T}
$$

where

$$
Z=\operatorname{Tr}_{E}\left\{e^{-H_{E} / T}\right\}
$$

is the partition function.

In (13) we have nonzero terms of the form

$$
\begin{aligned}
\operatorname{Tr}_{E}\left\{\rho_{E} A_{k}^{\dagger}(t) A_{l}(t-\tau)\right\} & =\frac{1}{Z} \operatorname{Tr}_{E}\left\{\exp \left[-\frac{1}{2 T} \sum_{j} \omega_{j} N_{j}\right] e^{-\iota \omega_{k} t} A_{k}^{\dagger} A_{l} e^{\iota \omega_{l}(t-\tau)}\right\} \\
& =\delta_{k, l} \frac{1}{Z} \sum_{n_{k}}\left[n_{k}\right]_{q} \exp \left[-\frac{\omega_{k}\left[n_{k}\right]_{q}}{2 T}\right] e^{-\imath \omega_{k} \tau}
\end{aligned}
$$


Here we have defined

$$
[n]_{q}=\frac{1-q^{n}}{1-q}
$$

as the $q$-deformed number.

Then, neglecting principal values terms, we obtain from (18)

$$
\begin{aligned}
\int_{0}^{\infty} d \tau \sum_{k, l} \lambda_{k} \lambda_{l} \operatorname{Tr}_{E}\left\{\rho_{E} A_{k}^{\dagger}(t) A_{l}(t-\tau)\right\} e^{\iota \Omega \tau} & =\int_{0}^{\infty} d \tau \sum_{k} \lambda_{k}^{2}\left\langle\left[N\left(\omega_{k}\right)\right]_{q}\right\rangle_{E} e^{-\iota\left(\omega_{k}-\Omega\right) \tau} \\
& =\sum_{k} \lambda_{k}^{2}\left\langle\left[N\left(\omega_{k}\right)\right]_{q}\right\rangle_{E} \delta\left(\omega_{k}-\Omega\right),
\end{aligned}
$$

where

$$
\left\langle\left[N\left(\omega_{k}\right)\right]_{q}\right\rangle_{E}=\frac{1}{Z} \sum_{n_{k}}\left[n_{k}\right]_{q} \exp \left[-\frac{\omega_{k}\left[n_{k}\right]_{q}}{2 T}\right] .
$$

Moving to the continuum of frequencies for the environment oscillators, we have

$$
\sum_{k} \lambda_{k}^{2}\left\langle\left[N\left(\omega_{k}\right)\right]_{q}\right\rangle_{E} \delta\left(\omega_{k}-\Omega\right) \rightarrow \int d \omega \Lambda^{2}(\omega)\left\langle[N(\omega)]_{q}\right\rangle_{E} \delta(\omega-\Omega)
$$

where $\Lambda^{2}(\omega)$ accounts for the coupling spectrum as well as for the density of states. As usual, we set $\Lambda^{2}(\Omega)=\gamma / 2$ to be the damping rate. Moreover, we get the following distribution [7, 8]

$$
\begin{aligned}
\left\langle[N]_{q}\right\rangle_{E} \equiv\left\langle[N(\Omega)]_{q}\right\rangle_{E} & =\frac{1}{e^{\Omega / T}-q}, \\
\left\langle[N+1]_{q}\right\rangle_{E} \equiv\left\langle[N(\Omega)+1]_{q}\right\rangle_{E} & =\frac{e^{\Omega / T}}{e^{\Omega / T}-q} .
\end{aligned}
$$

In summary, from (20), we have

$$
\int d \tau \sum_{k, l} \lambda_{k} \lambda_{l} \operatorname{Tr}_{E}\left\{\rho_{E} A_{k}^{\dagger}(t) A_{l}(t-\tau)\right\} e^{-\iota\left(\omega_{k}-\Omega\right) t+\iota\left(\omega_{l}-\Omega\right)(t-\tau)}=\frac{\gamma}{2}\left\langle[N]_{q}\right\rangle_{E} .
$$

Other nonzero terms in (13) are

$$
\int d \tau \sum_{k, l} \lambda_{k} \lambda_{l} \operatorname{Tr}_{E}\left\{\rho_{E} A_{k}(t) A_{l}^{\dagger}(t-\tau)\right\} e^{\iota\left(\omega_{k}-\Omega\right) t-\iota\left(\omega_{l}-\Omega\right)(t-\tau)}=\frac{\gamma}{2}\left\langle[N+1]_{q}\right\rangle_{E} .
$$

We finally arrive at the following master equation for the reduced system (the qubit):

$$
\dot{\rho}(t)=-\frac{\gamma}{2}\left\langle[N]_{q}\right\rangle_{E}\left(\sigma \sigma^{\dagger} \rho(t)-2 \sigma^{\dagger} \rho(t) \sigma+\rho(t) \sigma \sigma^{\dagger}\right)-\frac{\gamma}{2}\left\langle[N+1]_{q}\right\rangle_{E}\left(\sigma^{\dagger} \sigma \rho(t)-2 \sigma \rho(t) \sigma^{\dagger}+\rho(t) \sigma^{\dagger} \sigma\right) .
$$

This equation explicitly shows that the effect of the q-deformation is to change the rates of emission, which is proportional to $\left\langle[N]_{q}\right\rangle_{E}$, and the rate of absorption, proportional to $\left\langle[N+1]_{q}\right\rangle_{E}$ ( see also [8]). Notice that for $T=0$, there are no effects coming from the deformation, because for $N=0$ we simply have $\left\langle[0]_{q}\right\rangle_{E}=0$ and $\left\langle[1]_{q}\right\rangle_{E}=1$; in other words, the nonlinear effects introduced by the q-deformation cannot be observed if the environment transitions only concern the vacuum and the states with single excitation.

\section{ONE QUBIT}

Let us consider the operators appearing in Eq. (27) and represent them in matrix form in the computational basis $\{|0\rangle,|1\rangle\}$,

$$
\sigma=\left(\begin{array}{ll}
0 & 0 \\
1 & 0
\end{array}\right)
$$




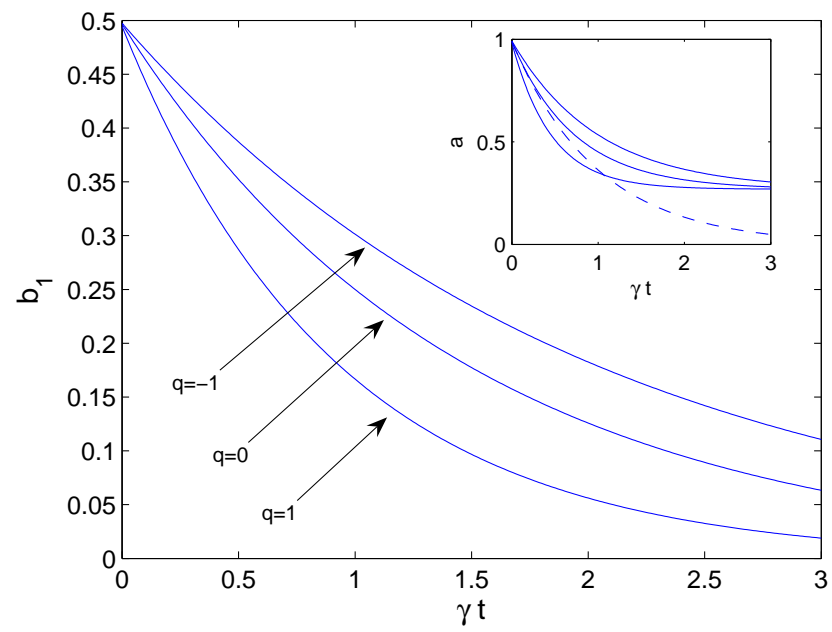

FIG. 1: The plot shows the decay of the coherence $b_{1}(t)$ for a qubit in a quon-environment at temperature $T / \Omega=1$, for different values of the deformation parameter. In the inset, it is shown the decay of the population $a(t)$ (solid lines refer again to $T / \Omega=1$, dashed line refers $T=0)$.

and

$$
\rho(t)=\left(\begin{array}{cc}
a(t) & b_{1}(t)+\iota b_{2}(t) \\
b_{1}(t)-\iota b_{2}(t) & 1-a(t)
\end{array}\right)
$$

where $a(t), b_{1}(t)$ and $b_{2}(t)$ are real functions of time to be determined.

Inserting the above matrices into Eq.(27) we get the following set of differential equations

$$
\begin{aligned}
\frac{d}{d t} a & =-2(A+B) a+2 A, \\
\frac{d}{d t}\left(b_{1}+\iota b_{2}\right) & =-(A+B)\left(b_{1}+\iota b_{2}\right),
\end{aligned}
$$

where for the sake of simplicity we have set

$$
\begin{aligned}
& A=(\gamma / 2)\left\langle[N]_{q}\right\rangle_{E}, \\
& B=(\gamma / 2)\left\langle[N+1]_{q}\right\rangle_{E} .
\end{aligned}
$$

The solutions of the differential equations (29), (30) read

$$
\begin{aligned}
a(t) & =\frac{e^{-2(A+B) t}}{A+B}\left[a(0) B+A\left(a(0)+e^{2(A+B) t}-1\right)\right], \\
b_{1}(t) & =b_{1}(0) e^{-(A+B) t} \\
b_{2}(t) & =b_{2}(0) e^{-(A+B) t} .
\end{aligned}
$$

Figure 1 shows the decay of the coherence $\left(b_{1}(t)\right)$ for a qubit in a quon environment at temperature $T / \Omega=1$, for different values of the deformation parameter. From Eq.s (31), (32), (34), it follows that the decay of coherence at $q=-1$, and any finite temperature, behaves as the decay at $T=0$ and any $q$. In the inset, it is shown the decay of the population $a(t)$ (solid lines refer again to $T / \Omega=1$, while dashed line refers $T=0$ ). Thus, the fermionic environment gives rise to the slowest decay of coherence and population. The decay of quantum coherence becomes slower and slower when passing from the bosonic to fermionic environment. 


\section{TWO QUBITS IN THE SAME ENVIRONMENT}

We now assume the system composed by two identical qubits interacting with the same environment. Then the master equation can be written as Eq.(27) simply replacing $\sigma$ with $\sigma_{1}+\sigma_{2}$, that is

$$
\begin{aligned}
& \dot{\rho}(t)=-\frac{\gamma}{2}\left\langle[N]_{q}\right\rangle_{E}\left(\sigma_{1} \sigma_{1}^{\dagger} \rho(t)-2 \sigma_{1}^{\dagger} \rho(t) \sigma_{1}+\rho(t) \sigma_{1} \sigma_{1}^{\dagger}+\sigma_{2} \sigma_{2}^{\dagger} \rho(t)\right. \\
&- 2 \sigma_{2}^{\dagger} \rho(t) \sigma_{2}+\rho(t) \sigma_{2} \sigma_{2}^{\dagger}+\sigma_{1} \sigma_{2}^{\dagger} \rho(t)+\sigma_{2} \sigma_{1}^{\dagger} \rho(t) \\
&\left.-2 \sigma_{1}^{\dagger} \rho(t) \sigma_{2}-2 \sigma_{2}^{\dagger} \rho(t) \sigma_{1}+\rho(t) \sigma_{1} \sigma_{2}^{\dagger}+\rho(t) \sigma_{2} \sigma_{1}^{\dagger}\right) \\
&-\frac{\gamma}{2}\left\langle[N+1]_{q}\right\rangle_{E}\left(\sigma_{1}^{\dagger} \sigma_{1} \rho(t)-2 \sigma_{1} \rho(t) \sigma_{1}^{\dagger}+\rho(t) \sigma_{1}^{\dagger} \sigma_{1}+\sigma_{2}^{\dagger} \sigma_{2} \rho(t)\right. \\
&-2 \sigma_{2} \rho(t) \sigma_{2}^{\dagger}+\rho(t) \sigma_{2}^{\dagger} \sigma_{2}+\sigma_{1}^{\dagger} \sigma_{2} \rho(t)+\sigma_{2}^{\dagger} \sigma_{1} \rho(t) \\
&\left.-2 \sigma_{1} \rho(t) \sigma_{2}^{\dagger}-2 \sigma_{2} \rho(t) \sigma_{1}^{\dagger}+\rho(t) \sigma_{1}^{\dagger} \sigma_{2}+\rho(t) \sigma_{2}^{\dagger} \sigma_{1}\right) .
\end{aligned}
$$

Then, we proceed in the same way as for the single qubit case. That is, we consider the operators appearing in Eq. (36) and represent them in matrix form in the computational basis $\{|00\rangle,|01\rangle|10\rangle,|11\rangle\}$,

$$
\sigma_{1}=\left(\begin{array}{cccc}
0 & 0 & 0 & 0 \\
0 & 0 & 0 & 0 \\
1 & 0 & 0 & 0 \\
0 & 1 & 0 & 0
\end{array}\right), \quad \sigma_{2}=\left(\begin{array}{llll}
0 & 0 & 0 & 0 \\
1 & 0 & 0 & 0 \\
0 & 0 & 0 & 0 \\
0 & 0 & 1 & 0
\end{array}\right),
$$

and

$$
\rho(t)=\left(\begin{array}{cccc}
a(t) & b_{1}(t)+\iota b_{2}(t) & c_{1}(t)+\iota c_{2}(t) & d_{1}(t)+\iota d_{2}(t) \\
b_{1}(t)-\iota b_{2}(t) & e(t) & f_{1}(t)+\iota f_{2}(t) & g_{1}(t)+\iota g_{2}(t) \\
c_{1}(t)-\iota c_{2}(t) & f_{1}(t)-\iota f_{2}(t) & h(t) & i_{1}(t)+\iota i_{2}(t) \\
d_{1}(t)-\iota d_{2}(t) & g_{1}(t)-\iota g_{2}(t) & i_{1}(t)-\iota i_{2}(t) & 1-a(t)-e(t)-h(t)
\end{array}\right)
$$

where $a(t), b_{1}(t), b_{2}(t), c_{1}(t), c_{2}(t), d_{1}(t), d_{2}(t), e(t), f_{1}(t), f_{2}(t), g_{1}(t), g_{2}(t), h(t), i_{1}(t)$ and $i_{2}(t)$ are real functions of time to be determined. In terms of these functions, the master equation is written as a set of coupled differential equations. They are reported together with their solutions in Appendix $\mathrm{A}$

In this case, a relevant quantity to study is the entanglement between the two qubits. Specifically we consider the qubits initialized in one of the four Bell states

$$
\begin{aligned}
& \left|\phi_{ \pm}\right\rangle=2^{-1 / 2}(|00\rangle \pm|11\rangle) \\
& \left|\psi_{ \pm}\right\rangle=2^{-1 / 2}(|01\rangle \pm|10\rangle)
\end{aligned}
$$

and then we investigate how entanglement decays.

We use the concurrence as measure of the degree of entanglement [9]

$$
C(\rho(t))=\max \left\{0, \lambda_{1}(t)-\lambda_{2}(t)-\lambda_{3}(t)-\lambda_{4}(t)\right\},
$$

where $\lambda_{i}(t)$ 's are, in decreasing order, the nonnegative square roots of the moduli of the eigenvalues of $\rho(t) \tilde{\rho}(t)$ with

$$
\tilde{\rho}(t)=\left(\sigma_{1}-\sigma_{1}^{\dagger}\right)\left(\sigma_{2}-\sigma_{2}^{\dagger}\right) \rho^{*}(t)\left(\sigma_{1}-\sigma_{1}^{\dagger}\right)\left(\sigma_{2}-\sigma_{2}^{\dagger}\right),
$$

and $\rho^{*}(t)$ denotes the complex conjugate of $\rho(t)$.

The decay of the concurrence is plotted in Figure 2 The qubits are initialized in the Bell states (39). For $T / \Omega=0$, the decay of the concurrence is independent from the deformation parameter $q$. For $T / \Omega>0$ we see the phenomenon of entanglement sudden death [10]. We notice however that the entanglement death time depends on the value of the deformation parameter $q$. In particular, the slowest decay and the longest lifetime of entanglement is evident for the fermionic case $q=-1$. The same happens when the two-qubit state is initialized in $\left|\psi_{+}\right\rangle=2^{-1 / 2}(|01\rangle+|10\rangle)$ (see inset). The decay of concurrence is slower and slower when continuously passing from the bosonic to the fermionic environment. On the contrary, the Bell state $\left|\psi_{-}\right\rangle$is invariant under the dynamics of (36), thus entanglement in this case is totally preserved. 


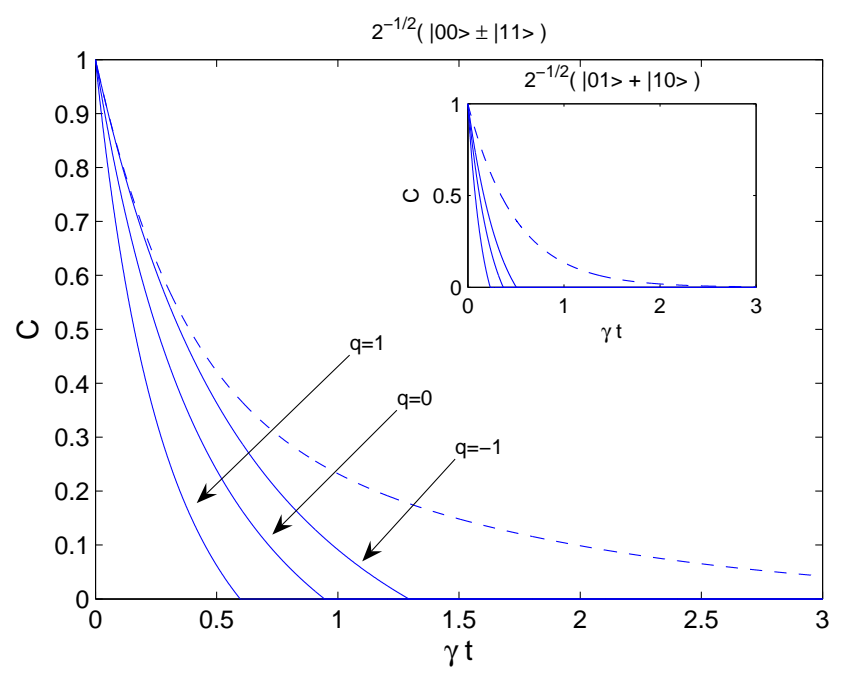

FIG. 2: The plot shows the decay of the concurrence for the two-qubit system in a quon-environment. Both qubits are plunged into the same environment. The qubits are initialized in one of the Bell states $\left|\phi_{ \pm}\right\rangle=2^{-1 / 2}(|00\rangle \pm|11\rangle)$; dashed line refer to $T / \Omega=0$, solid lines to $T / \Omega=1$ and several values of the deformation parameter $q$. In the inset the two-qubit state is initialized in the Bell state $\left|\psi_{+}\right\rangle=$ $2^{-1 / 2}(|01\rangle+|10\rangle)$. The remaining Bell state $\left|\psi_{-}\right\rangle=2^{-1 / 2}(|01\rangle-|10\rangle)$ is preserved by the dynamics.

\section{TWO QUBITS IN SEPARATE ENVIRONMENTS}

Here we consider each of the two identical qubit interacting with its own environment. Then the master equation is a straightforward extension of Eq.(27), that is

$$
\begin{aligned}
\dot{\rho}(t)= & -\frac{\gamma}{2}\left\langle[N]_{q}\right\rangle_{E}\left(\sigma_{1} \sigma_{1}^{\dagger} \rho(t)-2 \sigma_{1}^{\dagger} \rho(t) \sigma_{1}+\rho(t) \sigma_{1} \sigma_{1}^{\dagger}+\sigma_{2} \sigma_{2}^{\dagger} \rho(t)-2 \sigma_{2}^{\dagger} \rho(t) \sigma_{2}+\rho(t) \sigma_{2} \sigma_{2}^{\dagger}\right) \\
& -\frac{\gamma}{2}\left\langle[N+1]_{q}\right\rangle_{E}\left(\sigma_{1}^{\dagger} \sigma_{1} \rho(t)-2 \sigma_{1} \rho(t) \sigma_{1}^{\dagger}+\rho(t) \sigma_{1}^{\dagger} \sigma_{1}+\sigma_{2}^{\dagger} \sigma_{2} \rho(t)-2 \sigma_{2} \rho(t) \sigma_{2}^{\dagger}+\rho(t) \sigma_{2}^{\dagger} \sigma_{2}\right) .
\end{aligned}
$$

It can be solved with the same method of (36). The corresponding differential equations and their solutions are reported in AppendixB.

Figure 3 shows the decay of the concurrence in time. The qubits are initialized in the Bell states (39). For $T / \Omega=0$, the concurrence decay is independent from the deformation parameter $q$. Also in this case, for $T / \Omega>0$, we see the phenomenon of entanglement sudden death [10]. We notice that the entanglement death time depends on the value of the deformation parameter $q$. In particular, the slowest decay and the longest lifetime of entanglement is for the fermionic case $q=-1$. The same happens when the two-qubit state is initialized in $\left|\psi_{ \pm}\right\rangle=2^{-1 / 2}(|01\rangle \pm|10\rangle)$ (see inset). The decay of entanglement becomes slower and slower when passing from the bosonic to the fermionic environment. In this case there is no maximally entangled state that remains invariant under the dynamics.

\section{CONCLUSION}

In conclusion, we have analyzed the qubit dynamics in an environment of oscillators satisfying suitable $q$-deformed commutation relations, such that it permits to interpolate between oscillators and spin- $\frac{1}{2}$ particles. Specifically we have evaluated the decay of quantum coherence and entanglement in time when passing from bosonic to fermionic environments. The general behavior is that, at finite temperature, coherence and entanglement decay slower and slower when continuously passing from bosonic to fermionic environments.

Our work sheds further light on the mechanism of loosing quantum coherence and paves the way for a deeper algebraic analysis of this phenomenon. Moreover it could be useful for describing realistic physical situations where the assumption of an interaction with an environment of solely oscillators (resp. spin- $\frac{1}{2}$ ) particle turns out to be oversimplified.

\section{Acknowledgements}

The work of C.L. and S.M. is partially supported by EU through the FET-Open Project HIP (FP7-ICT-221899). 


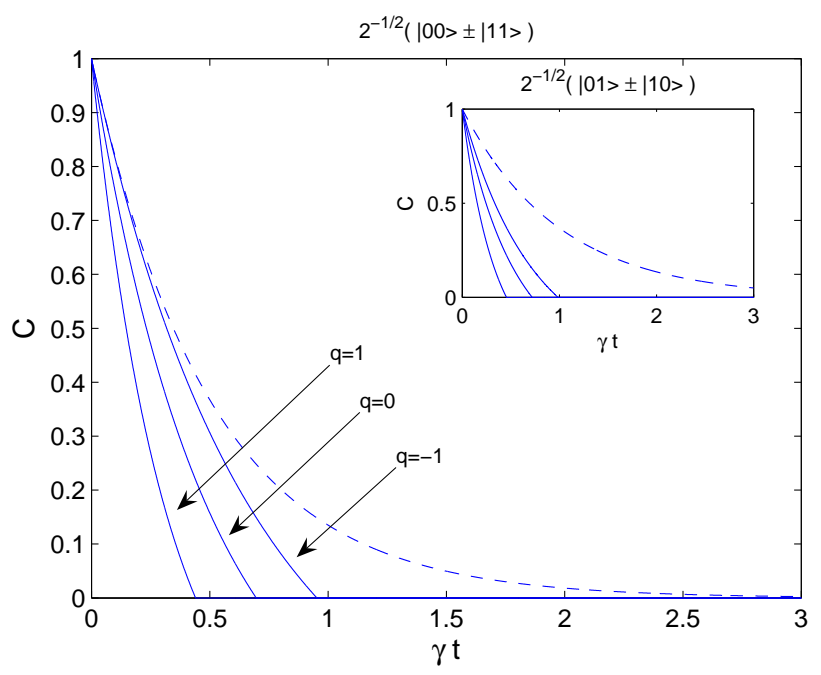

FIG. 3: The plot shows the decay of the concurrence of the two-qubit system in a quon-environment. Each qubit is subject to independent and identical environment. The qubits are initialized in one of the Bell states $\left|\phi_{ \pm}\right\rangle=2^{-1 / 2}(|00\rangle \pm|11\rangle)$; dashed line refer to $T / \Omega=0$, solid lines to $T / \Omega=1$ and several values of the deformation parameter $q$. In the inset the two-qubit state is initialized in one of the Bell states $\left|\psi_{ \pm}\right\rangle=2^{-1 / 2}(|01\rangle \pm|10\rangle)$

\section{APPENDIX A: TWO QUBITS IN THE SAME ENVIRONMENT}

Using the parametrization in (38), the master equation (36) translates in the following set of differential equations

$$
\begin{aligned}
\frac{d}{d t} a= & -4 B a+2 A\left(e+2 f_{1}+h\right) \\
\frac{d}{d t}\left(b_{1}+\iota b_{2}\right)= & -B\left(3 b_{1}+3 \iota b_{2}+c_{1}+\iota c_{2}\right) \\
& -A\left(b_{1}+\iota b_{2}+c_{1}+\iota c_{2}-2 g_{1}-2 \iota g_{2}-2 i_{1}-2 \iota i_{2}\right) \\
\frac{d}{d t}\left(c_{1}+\iota c_{2}\right)= & -B\left(b_{1}+\iota b_{2}+3 c_{1}+3 \iota c_{2}\right) \\
& -A\left(b_{1}+\iota b_{2}+c_{1}+\iota c_{2}-2 g_{1}-2 \iota g_{2}-2 i_{1}-2 \iota i_{2}\right) \\
\frac{d}{d t}\left(d_{1}+\iota d_{2}\right)= & -2(A+B)\left(d_{1}+\iota d_{2}\right) \\
\frac{d}{d t} e= & 2 B\left(a-e-f_{1}\right)+A\left(a+2 e+h+f_{1}-1\right) \\
\frac{d}{d t}\left(f_{1}+\iota f_{2}\right)= & B\left(2 a-e-h-2 f_{1}-2 \iota f_{2}\right) \\
& +A\left(2-2 a-3 e-3 h-2 f_{1}-2 \iota f_{2}\right) \\
\frac{d}{d t}\left(g_{1}+\iota g_{2}\right)= & B\left(2 b_{1}+2 \iota b_{2}+2 c_{1}+2 \iota c_{2}-g_{1}-\iota g_{2}-i_{1}-\iota i_{2}\right) \\
& -A\left(3 g_{1}+3 \iota g_{2}+i_{1}+\iota i_{2}\right) \\
\frac{d}{d t} h= & 2 B\left(a-h-f_{1}\right)-2 A\left(a+e+2 h+f_{1}-1\right) \\
\frac{d}{d t}\left(i_{1}+\iota i_{2}\right)= & B\left(2 b_{1}+2 \iota b_{2}+2 c_{1}+2 \iota c_{2}-g_{1}-\iota g_{2}-i_{1}-\iota i_{2}\right) \\
& -A\left(g_{1}+\iota g_{2}+3 i_{1}+3 \iota i_{2}\right)
\end{aligned}
$$

The solutions of the differential equations (A1)-(A9) read 


$$
\begin{aligned}
& a(t)=\frac{1}{4 \sqrt{B}} e^{-4(\sqrt{A B}+B) t}\left[2 a(0) \sqrt{B}\left(1+e^{8 \sqrt{A B} t}\right)\right. \\
& \left.+\sqrt{A}\left(e^{8 \sqrt{A B} t}-1\right)\left(e(0)+2 f_{1}(0)+h(0)\right)\right] \text {, } \\
& b_{1}(t)=\frac{e^{-(3 A+3 B+\Theta) t}}{4 \Theta^{2}}\left\{A ^ { 2 } \left[c_{1}(0)\left(1+e^{2 \Theta t}-2 e^{(3 A+B+\Theta) t}\right)\right.\right. \\
& \left.+b_{1}(0)\left(1+e^{2 \Theta t}+2 e^{(3 A+B+\Theta) t}\right)\right]+B \Theta\left(b_{1}(0)+c_{1}(0)\right)\left(1-e^{2 \Theta t}\right) \\
& +B^{2}\left[+c_{1}(0)\left(1+e^{2 \Theta t}-2 e^{(3 A+B+\Theta) t}\right)+b_{1}(0)\left(1+e^{2 \Theta t}+2 e^{(3 A+B+\Theta) t}\right)\right] \\
& +A\left[14 B\left(c_{1}(0)\left(1+e^{2 \Theta t}-2 e^{(3 A+B+\Theta) t}\right)+b_{1}(0)\left(1+e^{2 \Theta t}+2 e^{(3 A+B+\Theta) t}\right)\right)\right. \\
& \left.\left.+\Theta\left(-1+e^{2 \Theta t}\right)\left(b_{1}(0)+c_{1}(0)+4 g_{1}(0)+4 i_{1}(0)\right)\right]\right\} \\
& c_{1}(t)=\frac{e^{-(3 A+3 B+\Theta) t}}{4 \Theta^{2}}\left\{A ^ { 2 } \left[b_{1}(0)\left(1+e^{2 \Theta t}-2 e^{(3 A+B+\Theta) t}\right)\right.\right. \\
& \left.+c_{1}(0)\left(1+e^{2 \Theta t}+2 e^{(3 A+B+\Theta) t}\right)\right]+B \Theta\left(b_{1}(0)+c_{1}(0)\right)\left(1-e^{2 \Theta t}\right) \\
& +B^{2}\left[b_{1}(0)\left(1+e^{2 \Theta t}-2 e^{(3 A+B+\Theta) t}\right)+c_{1}(0)\left(1+e^{2 \Theta t}+2 e^{(3 A+B+\Theta) t}\right)\right] \\
& +A\left[14 B\left(b_{1}(0)\left(1+e^{2 \Theta t}-2 e^{(3 A+B+\Theta) t}\right)+c_{1}(0)\left(1+e^{2 \Theta t}+2 e^{(3 A+B+\Theta) t}\right)\right)\right. \\
& \left.\left.+\Theta\left(-1+e^{2 \Theta t}\right)\left(b_{1}(0)+c_{1}(0)+4 g_{1}(0)+4 i_{1}(0)\right)\right]\right\} \text {, } \\
& d_{1}(t)=d_{1}(0) e^{-2(A+B) t}, \\
& e(t)=\frac{e^{-2(3 A+2 \sqrt{A B}+3 B) t}}{8 \sqrt{A B}\left(A^{2}-3 A B+B^{2}\right)}\left\{-2 a(0) A B^{2} e^{2(3 A+B) t}\left(e^{8 \sqrt{A B} t}-1\right)\right. \\
& +2 a(0) B^{3} e^{2(3 A+B) t}\left(e^{8 \sqrt{A B} t}-1\right) \\
& -A^{3} e^{2(3 A+B) t}\left(e^{8 \sqrt{A B} t}-1\right)\left(e(0)+2 f_{1}(0)+h(0)\right) \\
& +3 A^{2} B e^{2(3 A+B) t}\left(e^{8 \sqrt{A B} t}-1\right)\left(e(0)+2 f_{1}(0)+h(0)\right) \\
& +\sqrt{A B^{5}} e^{2(A+B) t}\left[4 e^{4(A+\sqrt{A B}+B) t}+4 e^{2(\sqrt{A}+\sqrt{B})^{2} t}(e(0)-h(0))\right. \\
& +2 e^{4 \sqrt{A B} t+4 B t}\left(e(0)-2 f_{1}(0)+h(0)-2\right) \\
& \left.+e^{4 A t}\left(e(0)+2 f_{1}(0)+h(0)\right)+e^{4 A t+8 \sqrt{A B} t}\left(e(0)+2 f_{1}(0)+h(0)\right)\right] \\
& -\sqrt{A^{3} B^{3}} e^{2(A+B) t}\left[12 e^{4(A+\sqrt{A B}+B) t}+12 e^{2(\sqrt{A}+\sqrt{B})^{2} t}(e(0)-h(0))\right. \\
& -6 a(0)\left(e^{4 A t}+e^{4 A t+8 \sqrt{A B} t}-2 e^{4 \sqrt{A B} t+4 B t}\right)+e^{4 A t}\left(e(0)+2 f_{1}(0)+h(0)\right) \\
& +e^{4 A t+8 \sqrt{A B} t}\left(e(0)+2 f_{1}(0)+h(0)\right) \\
& \left.+2 e^{4 \sqrt{A B} t+4 B t}\left(5 e(0)-2 f_{1}(0)+5 h(0)-6\right)\right] \\
& +2 \sqrt{A^{5} B}\left[-a(0)\left(e^{2(3 A+B) t}+e^{2(3 A+4 \sqrt{A B}+B) t}-2 e^{2(A+2 \sqrt{A B}+3 B) t}\right)\right. \\
& +2 e^{2(A+2 \sqrt{A B}+2 B) t}\left(e^{2(2 A+B) t}+e^{2 A t}(e(0)-h(0))\right. \\
& \left.\left.\left.+e^{2 B t}(e(0)+h(0)-1)\right)\right]\right\},
\end{aligned}
$$




$$
\begin{aligned}
& f_{1}(t)=\frac{e^{-4(A+\sqrt{A B}+B) t}}{8 \sqrt{A B}\left(A^{2}-3 A B+B^{2}\right)}\left\{-10 a(0) A B^{2} e^{4 A t}\left(e^{8 \sqrt{A B} t}-1\right)\right. \\
& +2 a(0) B^{3} e^{4 A t}\left(e^{8 \sqrt{A B} t}-1\right)+A^{3} e^{4 A t}\left(e^{8 \sqrt{A B} t}-1\right)\left(e(0)+2 f_{1}(0)+h(0)\right) \\
& +A^{2} B e^{4 A t}\left(e^{8 \sqrt{A B} t}-1\right)\left(4 a(0)-3 e(0)-6 f_{1}(0)-3 h(0)\right) \\
& -2 \sqrt{A^{5} B}\left[2 e^{4(A+\sqrt{A B}+B) t}-a(0)\left(e^{4 A t}-2 e^{4(\sqrt{A B}+B) t}+e^{4 A t+8 \sqrt{A B} t}\right)\right. \\
& +2 e^{4(\sqrt{A B}+B) t}(e(0)+h(0)-1) \\
& \left.-e^{4 A t}\left(e(0)+2 f_{1}(0)+h(0)\right)-e^{4 A t+8 \sqrt{A B} t}\left(e(0)+2 f_{1}(0)+h(0)\right)\right] \\
& +\sqrt{A B^{5}}\left[-4 e^{4(A+\sqrt{A B}+B) t}-2 e^{4(\sqrt{A B}+B) t}\left(e(0)-2 f_{1}(0)+h(0)-2\right)\right. \\
& \left.+e^{4 A t}\left(e(0)+2 f_{1}(0)+h(0)\right)+e^{4 A t+8 \sqrt{A B} t}\left(e(0)+2 f_{1}(0)+h(0)\right)\right] \\
& +\sqrt{A^{3} B^{3}}\left[12 e^{4(A+\sqrt{A B}+B) t}-6 a(0)\left(e^{4 A t}-2 e^{4(\sqrt{A B}+B) t}+e^{4 A t+8 \sqrt{A B} t}\right)\right. \\
& -5 e^{4 A t}\left(e(0)+2 f_{1}(0)+h(0)\right)-5 e^{4 A t+8 \sqrt{A B} t}\left(e(0)+2 f_{1}(0)+h(0)\right) \\
& \left.\left.+2 e^{4(\sqrt{A B}+B) t}\left(5 e(0)-2 f_{1}(0)+5 h(0)-6\right)\right]\right\} \\
& f_{2}(t)=f_{2}(0) e^{-2(A+B) t} \\
& g_{1}(t)=\frac{e^{-(3 A+3 B+\Theta) t}}{4 \Theta^{2}}\left\{B \left[4 \Theta\left(b_{1}(0)+c_{1}(0)\right)\left(-1+e^{2 \Theta t}\right)+14 A\left(g_{1}(0)+i_{1}(0)\right)\right.\right. \\
& -\Theta\left(g_{1}(0)+i_{1}(0)\right)+\Theta e^{2 \Theta t}\left(g_{1}(0)+i_{1}(0)\right) \\
& \left.+28 A e^{(A+3 B+\Theta) t}\left(g_{1}(0)-i_{1}(0)\right)+14 A e^{2 \Theta t}\left(g_{1}(0)+i_{1}(0)\right)\right] \\
& +B^{2}\left[\left(1+e^{2 \Theta t}+2 e^{(A+3 B+\Theta) t}\right) g_{1}(0)+\left(1+e^{2 \Theta t}-2 e^{(A+3 B+\Theta) t}\right) i_{1}(0)\right] \\
& +A\left[\Theta\left(1-e^{2 \Theta t}\right)\left(g_{1}(0)+i_{1}(0)\right)+A\left(\left(1+e^{2 \Theta t}+2 e^{(A+3 B+\Theta) t}\right) g_{1}(0)\right.\right. \\
& \left.\left.\left.+\left(1+e^{2 \Theta t}-2 e^{(A+3 B+\Theta) t}\right) i_{1}(0)\right)\right]\right\}
\end{aligned}
$$




$$
\begin{aligned}
& h(t)=\frac{e^{-2(3 A+2 \sqrt{A B}+3 B) t}}{8 \sqrt{A B}\left(A^{2}-3 A B+B^{2}\right)}\left\{-2 a(0) A B^{2} e^{2(3 A+B) t}\left(e^{8 \sqrt{A B} t}-1\right)\right. \\
& +2 a(0) B^{3} e^{2(3 A+B) t}\left(e^{8 \sqrt{A B} t}-1\right) \\
& -A^{3} e^{2(3 A+B) t}\left(e^{8 \sqrt{A B} t}-1\right)\left(e(0)+2 f_{1}(0)+h(0)\right) \\
& +3 A^{2} B e^{2(3 A+B) t}\left(e^{8 \sqrt{A B} t}-1\right)\left(e(0)+2 f_{1}(0)+h(0)\right) \\
& +\sqrt{A B^{5}} e^{2(A+B) t}\left[4 e^{4(A+\sqrt{A B}+B) t}-4 e^{2(\sqrt{A}+\sqrt{B})^{2} t}(e(0)-h(0))\right. \\
& +2 e^{4 \sqrt{A B} t+4 B t}\left(e(0)-2 f_{1}(0)+h(0)-2\right) \\
& \left.+e^{4 A t}\left(e(0)+2 f_{1}(0)+h(0)\right)+e^{4 A t+8 \sqrt{A B} t}\left(e(0)+2 f_{1}(0)+h(0)\right)\right] \\
& -\sqrt{A^{3} B^{3}} e^{2(A+B) t}\left[12 e^{4(A+\sqrt{A B}+B) t}\right. \\
& -6 a(0)\left(e^{4 A t}+e^{4 A t+8 \sqrt{A B} t}-2 e^{4 \sqrt{A B} t+4 B t}\right) \\
& -12 e^{2(\sqrt{A}+\sqrt{B})^{2} t}(e(0)-h(0))+e^{4 A t}\left(e(0)+2 f_{1}(0)+h(0)\right) \\
& +e^{4 A t+8 \sqrt{A B} t}\left(e(0)+2 f_{1}(0)+h(0)\right) \\
& \left.+2 e^{4 \sqrt{A B} t+4 B t}\left(5 e(0)-2 f_{1}(0)+5 h(0)-6\right)\right] \\
& +2 \sqrt{A^{5} B}\left[-a(0)\left(e^{2(3 A+B) t}+e^{2(3 A+4 \sqrt{A B}+B) t}-2 e^{2(A+2 \sqrt{A B}+3 B) t}\right)\right. \\
& +2 e^{2(A+2 \sqrt{A B}+2 B) t}\left(e^{2(2 A+B) t}-e^{2 A t}(e(0)-h(0))\right. \\
& \left.\left.\left.+e^{2 B t}(e(0)+h(0)-1)\right)\right]\right\} \\
& i_{1}(t)=\frac{e^{-(3 A+3 B+\Theta) t}}{4 \Theta^{2}}\left\{B \left[4 \Theta\left(b_{1}(0)+c_{1}(0)\right)\left(-1+e^{2 \Theta t}\right)\right.\right. \\
& +14 A\left(g_{1}(0)+i_{1}(0)\right)-\Theta\left(g_{1}(0)+i_{1}(0)\right)+\Theta e^{2 \Theta t}\left(g_{1}(0)+i_{1}(0)\right) \\
& \left.-28 A e^{(A+3 B+\Theta) t}\left(g_{1}(0)-i_{1}(0)\right)+14 A e^{2 \Theta t}\left(g_{1}(0)+i_{1}(0)\right)\right] \\
& +B^{2}\left[\left(1+e^{2 \Theta t}-2 e^{(A+3 B+\Theta) t}\right) g_{1}(0)+\left(1+e^{2 \Theta t}+2 e^{(A+3 B+\Theta) t}\right) i_{1}(0)\right] \\
& +A^{2}\left[\left(1+e^{2 \Theta t}-2 e^{(A+3 B+\Theta) t}\right) g_{1}(0)+\left(1+e^{2 \Theta t}+2 e^{(A+3 B+\Theta) t}\right) i_{1}(0)\right] \\
& \left.+A\left[\Theta\left(1-e^{2 \Theta t}\right)\left(g_{1}(0)+i_{1}(0)\right)\right]\right\},
\end{aligned}
$$

where $\Theta=\sqrt{A^{2}+14 A B+B^{2}}$. The other solutions $b_{2}(t), c_{2}(t), d_{2}(t), g_{2}(t)$ and $i_{2}(t)$ can be obtained from $b_{1}(t), c_{1}(t)$, $d_{1}(t), g_{1}(t)$ and $i_{1}(t)$ respectively by simply replacing the subscripts $1 \rightarrow 2$. 


\section{APPENDIX B: TWO QUBITS IN SEPARATE ENVIRONMENTS}

Using the parametrization in (38), the master equation (43) translates in the following set of differential equations

$$
\begin{aligned}
\frac{d}{d t} a & =-4 B a+2 A(e+h), \\
\frac{d}{d t}\left(b_{1}+\iota b_{2}\right) & =-3 B\left(b_{1}+\iota b_{2}\right)-A\left(b_{1}+\iota b_{2}-2 i_{1}-2 \iota i_{2}\right), \\
\frac{d}{d t}\left(c_{1}+\iota c_{2}\right) & =-3 B\left(c_{1}+\iota c_{2}\right)-A\left(c_{1}+\iota c_{2}-2 g_{1}-2 \iota g_{2}\right), \\
\frac{d}{d t}\left(d_{1}+\iota d_{2}\right) & =-2(A+B)\left(d_{1}+\iota d_{2}\right), \\
\frac{d}{d t} e & =2 B(a-e)-2 A(a+2 e+h-1), \\
\frac{d}{d t}\left(f_{1}+\iota f_{2}\right) & =-2(A+B)\left(f_{1}+\iota f_{2}\right), \\
\frac{d}{d t}\left(g_{1}+\iota g_{2}\right) & =B\left(2 c_{1}+2 \iota c_{2}-g_{1}-\iota g_{2}\right)-3 A\left(g_{1}+\iota g_{2}\right), \\
\frac{d}{d t} h & =2 B(a-h)-2 A(a+e+2 h-1), \\
\frac{d}{d t}\left(i_{1}+\iota i_{2}\right) & =B\left(2 b_{1}+2 \iota b_{2}-i_{1}-\iota i_{2}\right)-3 A\left(i_{1}+\iota i_{2}\right) .
\end{aligned}
$$

The solutions of the differential equations (B1)-(B9) read

$$
\begin{aligned}
& a(t)=\frac{e^{-4(A+B) t}}{(A+B)^{2}}\left\{a(0) B^{2}+A^{2}\left[a(0)\left(2 e^{2(A+B) t}-1\right)\right.\right. \\
& \left.+\left(e^{2(A+B) t}-1\right)\left(e^{2(A+B) t}+e(0)+h(0)-1\right)\right] \\
& \left.+\left[2 a(0) e^{2(A+B) t}+(e(0)+h(0))\left(e^{2(A+B) t}-1\right)\right] A B\right\}, \\
& b_{1}(t)=\frac{e^{-3(A+B) t}}{A+B}\left\{b_{1}(0) B+A\left[b_{1}(0) e^{2(A+B) t}+\left(e^{2(A+B) t}-1\right) i_{1}(0)\right]\right\} \text {, } \\
& c_{1}(t)=\frac{e^{-3(A+B) t}}{A+B}\left\{c_{1}(0) B+A\left[c_{1}(0) e^{2(A+B) t}+\left(e^{2(A+B) t}-1\right) g_{1}(0)\right]\right\}, \\
& d_{1}(t)=d_{1}(0) e^{-2(A+B) t}, \\
& e(t)=\frac{e^{-4(A+B) t}}{(A+B)^{2}}\left\{\left[a(0)\left(1-e^{2(A+B) t}\right)+e(0)-e^{2(A+B) t}(h(0)-1)+h(0)-1\right] A^{2}\right. \\
& +\left[e(0)+e^{4(A+B) t}+e^{2(A+B) t}(e(0)-h(0)-1)+h(0)\right] A B \\
& \left.+\left[a(0)\left(e^{2(A+B) t}-1\right)+e(0) e^{2(A+B) t}\right] B^{2}\right\} \\
& f_{1}(t)=f_{1}(0) e^{-2(A+B) t}, \\
& g_{1}(t)=\frac{e^{-3(A+B) t}}{A+B}\left\{g_{1}(0) B+A\left[g_{1}(0) e^{2(A+B) t}+\left(e^{2(A+B) t}-1\right) c_{1}(0)\right]\right\} \text {, } \\
& h(t)=\frac{e^{-4(A+B) t}}{(A+B)^{2}}\left\{\left[a(0)\left(1-e^{2(A+B) t}\right)-e^{2(A+B) t}(e(0)-1)+e(0)+h(0)-1\right] A^{2}\right. \\
& +\left[e(0)+e^{4(A+B) t}+h(0)+e^{2(A+B) t}(h(0)-e(0)-1)\right] A B \\
& \left.+\left[a(0)\left(e^{2(A+B) t}-1\right)+h(0) e^{2(A+B) t}\right] B^{2}\right\} \\
& i_{1}(t)=\frac{e^{-3(A+B) t}}{A+B}\left\{i_{1}(0) B+A\left[i_{1}(0) e^{2(A+B) t}+\left(e^{2(A+B) t}-1\right) b_{1}(0)\right]\right\} .
\end{aligned}
$$


The other solutions $b_{2}(t), c_{2}(t), d_{2}(t), f_{2}(t), g_{2}(t)$ and $i_{2}(t)$ can be obtained from $b_{1}(t), c_{1}(t), d_{1}(t), f_{1}(t), g_{1}(t)$ and $i_{1}(t)$ respectively by simply replacing the subscripts $1 \rightarrow 2$.

[1] C. W. Gardiner, Quantum Noise, Springer, Berlin 1991.

[2] V. Kuryshkin, Annales de la Fondation Louis de-Broglie 5 (1980), 111.

[3] P. Kulish and E. Damaskinsky, J. Phys. A 23 (1990), L415.

[4] A. J. Macfarlane, J. Phys. A 22 (1989), 4581;

L. C. Biedenharn, J. Phys. A 22 (1989), L873;

C.-P. Sun and H. C. Fu, J. Phys. A 22 (1989), L983.

[5] O. W. Greenberg, Phys. Rev. Lett. 64 (1990), 705; Phys. Rev. D 43 (1991), 4111.

[6] R. N. Mohapatra, Phys. Lett. B 242 (1990), 407.

[7] M. Chaichian et al., J. Phys. A 26 (1993), 4017.

[8] J. W. Goodison and D. J. Toms arXiv:hep-th/9410096

[9] W. K. Wootters, Phys. Rev. Lett. 80 (1998), 2245.

[10] T. Yu and J. H. Eberly, Phys. Rev. Lett. 93 (2004), 140404;

A. Al-Qasimi and D. F. V. James, Phys. Rev. A 77 (2008), 012117. 